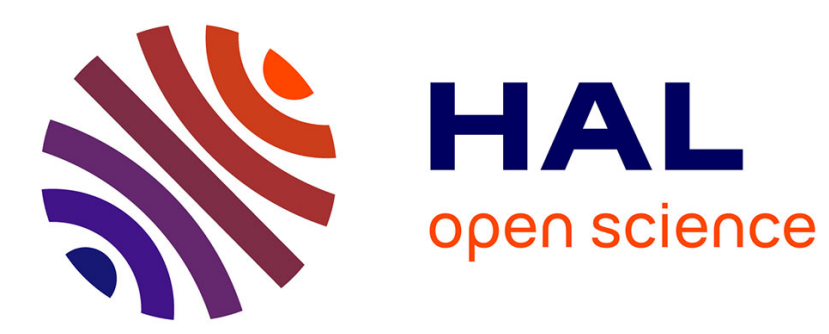

\title{
3-dimensional particulate flow modelling using a viscous penalty combined with a stable projection scheme
}

\author{
Léa Batteux, Jacques Laminie, Jean-Claude Latché, Pascal Poullet
}

\section{To cite this version:}

Léa Batteux, Jacques Laminie, Jean-Claude Latché, Pascal Poullet. 3-dimensional particulate flow modelling using a viscous penalty combined with a stable projection scheme. Finite Volumes for Complex Applications IX, Jun 2020, Bergen, Norway. hal-03089404

\section{HAL Id: hal-03089404 https://hal.science/hal-03089404}

Submitted on 28 Dec 2020

HAL is a multi-disciplinary open access archive for the deposit and dissemination of scientific research documents, whether they are published or not. The documents may come from teaching and research institutions in France or abroad, or from public or private research centers.
L'archive ouverte pluridisciplinaire HAL, est destinée au dépôt et à la diffusion de documents scientifiques de niveau recherche, publiés ou non, émanant des établissements d'enseignement et de recherche français ou étrangers, des laboratoires publics ou privés.

$$
\text { Copyright }
$$




\title{
3-dimensional particulate flow modelling using a viscous penalty combined with a stable projection scheme
}

\author{
L. Batteux, J. Laminie, J.C. Latché and P. Poullet
}

5 Abstract We introduce a strategy for the simulation a particulate flow in a 3dimensional domain. The particles are assumed to be rigid, and the homogeneous fluid flow to be governed by the incompressible Navier-Stokes equations. The system is solved using a predictor-corrector scheme for the Navier-Stokes equations with variable density. The latter scheme is adapted to take into account the solid domain by adopting a volume penalization method. In order to advect efficiently the particles, the approximation of the mass balance equation is carried out by an antidissipative scheme similar to the Ultra-Bee scheme. We conclude with numerical tests in the context of particulate flows.

Key words: $65 \mathrm{M} 08,76 \mathrm{D} 05,76 \mathrm{~T} 20$

\section{Introduction}

This work is focused on the modelling of fluid-solid systems in a 3-dimensional domain. To reproduce faithfully the fluid-solid interactions is a problem of large interest due to numerous processes in industrial applications. There are several methods to attempt to model such a problem, but in this study, one considers rigid solid inclusions in an incompressible viscous fluid flow and one enforces a strong coupling between both phases. The motion of the solid domain may then be described using Newton laws for rigid bodies. As we are concerned with the efficiency and computational costs, we resort to an Eulerian formulation for the fluid flow and extend the fluid problem inside the solid domain in the manner of fictitious domain

L. Batteux, J. Laminie, P. Poullet

LAMIA, Université des Antilles, Campus de Fouillole, Pointe-à-Pitre, 97157 Guadeloupe FWI

e-mail: lea.batteux@univ-antilles.fr, pascal.poullet@univ-antilles.fr

J.C. Latché

IRSN, BP13115, St-Paul-lez-Durance Cedex, France e-mail: jean-claude.latche@ irsn.fr 
methods $[1,11,3]$. In our case we enforce some kind of Brinkmann law inside the solid domain by adopting the H1-penalty method $[1,11]$. In practice, it will come down to penalizing the tensor of deformation term where the particles are in the nonhomogeneous Navier-Stokes equations. Let us denote by $\Omega$ the domain containing the particulate flow. The continuous problem is given by:

$$
\left\{\begin{array}{lr}
\frac{\partial \rho}{\partial t}+\nabla \cdot(\rho \mathbf{v})=0 & \text { in } \mathbb{R}^{+} \times \Omega \\
\frac{\partial(\rho \mathbf{v})}{\partial t}+\nabla \cdot(\rho \mathbf{v} \otimes \mathbf{v})-2 \nabla \cdot(\mu(\rho) \mathbf{D}(\mathbf{v}))+\nabla p=\mathbf{f} & \text { in } \mathbb{R}^{+} \times \Omega \\
\nabla \cdot \mathbf{v}=0 & \text { in } \mathbb{R}^{+} \times \Omega
\end{array}\right.
$$

30

The flow being incompressible, one often resorts to a projection scheme $[8,5]$ to solve problem (1). Many variants can be found in the litterature especially when considering multiphase flows. However a lot of problems remain open regarding the stability or convergence of those schemes. Again, for efficiency reasons we will rely on the scheme introduced in [9]. In this article, the authors circumvent the relatively expensive computational cost of the elliptic pressure problem of standard projection problem for incompressible variable density flows, by switching to an approximate and more efficient formulation of the latter. In this paper, we adapt the scheme presented in [9] for finite elements to the MAC discretization, while keeping the same stability properties. Additionally, the discontinuity and the jumps of viscosity along the fluid/solid interface require an accurate tracking of the surface of the particles.

so To this end, we replace the discrete mass balance equation with an anti-diffusive advection scheme introduced in [4] that is similar to the Ultra-Bee method. The scheme is adapted to the dimension $d=3$ by considering an alternate direction variant.

In the following section we introduce the notations, meshes as well as the full discrete scheme. In a final section we carry out and comment on the simulation of the fall of a rigid sphere. 


\section{Numerical Method}

Let $0=t_{0}<t_{1}<\cdots<t_{N}=T$ be a uniform partition of the time interval $[0, T]$. We note $\delta t=T / N$ the time step so that $t_{n}=n \delta t$, for $n \in \llbracket 0, N \rrbracket$. The incremental

projection scheme from [9] reads,

$$
\begin{gathered}
\frac{\rho^{n+1}-\rho^{n}}{\delta t}+\nabla \cdot\left(\rho^{n+1} \overline{\mathbf{v}}^{\mathbf{n}}\right)=0 \text { in } \Omega \\
\frac{\rho^{n+1} \mathbf{v}^{n+1}-\rho^{n} \mathbf{v}^{n}}{\delta t}+\nabla \cdot\left(\rho^{n+1} \mathbf{v}^{n+1} \otimes \overline{\mathbf{v}}^{\mathbf{n}}\right)-\nabla \cdot\left(\left(\mu^{n+1} D\left(\mathbf{v}^{n+1}\right)\right)\right) \\
+\nabla\left(2 p^{n}-p^{n-1}\right)=\mathbf{f}^{n+1} \text { in } \Omega \\
\mathbf{v}^{n+1}=0 \text { on } \partial \Omega \\
-\frac{\delta t}{\chi} \Delta\left(p^{n+1}-p^{n}\right)=-\nabla \cdot \mathbf{v}^{\mathbf{n}+\mathbf{1}} \text { in } \Omega \\
\mathbf{\mathbf { v }}^{\mathbf{n}+\mathbf{1}}=\mathbf{v}^{n+1}-\frac{\delta t}{\chi} \nabla\left(p^{n+1}-p^{n}\right) \text { in } \Omega
\end{gathered}
$$

given for any time increment $t^{n+1}$. We denote $\mathbf{f}\left(t^{n+1}\right)=\mathbf{f}^{n+1}$ and we take $\chi=\min _{\mathbf{x} \in \Omega} \rho_{0}$. We aim to compute the sequence of discrete solution $\left(\mathbf{v}^{n+1}, \overline{\mathbf{v}}^{n+1}, p^{n+1}, \rho^{n+1}\right)$ for $n \geq 0$. For each time step, we obtain a tentative velocity $\mathbf{v}^{n+1}$ by solving equation (2b). Using the Helmholtz decomposition of $\mathbf{L}^{2}(\Omega)$, the substep (2c) acts as the projection of $\mathbf{v}^{n+1}$ on $\mathbf{H}=\left\{\mathbf{v} \in \mathbf{L}^{2}(\Omega), \nabla \cdot \mathbf{v}=0, \mathbf{v} \cdot \mathbf{n}_{\partial \Omega \Omega}=0\right\}$ to get a corrected divergence-free velocity $\overline{\mathbf{v}}^{n+1}$ in (2d). The advection of the density is carried out by (2a). The specificity (and interest) of the present scheme is to replace the actual density by the constant coefficient $\chi$; indeed, a direct adaptation of the classic incremental projection scheme [8] to nonhomogeneous fluids would result in a variable Poisson problem of the form $\nabla \cdot\left(\left(\rho^{n+1}\right)^{-1} \nabla \Phi^{n+1}\right)=0$ with Neumann boundary conditions. For discontinuous densities with a high $\rho_{\max } / \rho_{\min }$ ratio, this problem can be expensive to solve due to its ill-conditioned status.

\subsection{Notations, mesh and discrete projection scheme}

The domain $\Omega$ is discretized according to a staggered MAC mesh $\mathscr{D}=(\mathscr{M}, \mathscr{E})$ so the scheme (2) can benefit from the infsup stability property. Let the primal grid $\mathscr{M}$ consists in a conforming structured partition of $\Omega$ using rectangular parallelepipeds elements. The parallelepipeds will be defined as primal cells and noted $K$. Therefore we have $\overline{\cup_{K \in \mathscr{M}} K}=\bar{\Omega}$. We may assume that the faces of the primal cells are normal to one vector of the standard basis of $\mathbb{R}^{3}$, denoted by $\left(\mathbf{e}_{1}, \ldots, \mathbf{e}_{d}\right)$. A face of the primal cell $K \in \mathscr{M}$ will be noted $\sigma \in \mathscr{E}(K), \mathscr{E}(K)$ referring to the set of all faces of $K$. 
The staggered grid is completed by defining the dual grid $\mathscr{E}$ as the set of all edges of $\mathscr{M}:\{\sigma \in \mathscr{E}(K) \mid K \in \mathscr{M}\}$. We note $\mathscr{E}=\mathscr{E}_{\text {int }} \cup \mathscr{E}_{\text {ext }}$, where $\mathscr{E}_{\text {int }}$ (resp. $\mathscr{E}_{\text {ext }}$ ) are the edges of $\mathscr{E}$ that lie in the interior (resp. on the boundary) of the domain. The set of faces that are orthogonal to the $i^{\text {th }}$ unit vector $\mathbf{e}_{i}$ of the canonical basis of $\mathbb{R}^{d}$ is denoted by $\mathscr{E}^{(i)}$, for $i=1, \ldots, d$. Correspondingly we introduce $\mathscr{E}_{\text {int }}^{(i)}=\mathscr{E}_{\text {int }} \cap \mathscr{E}^{(i)}$, $\mathscr{E}_{\text {ext }}^{(i)}=\mathscr{E}_{\text {ext }} \cap \mathscr{E}^{(i)}$ so $\mathscr{E}^{(i)}=\mathscr{E}_{\text {int }}^{(i)} \cup \mathscr{E}_{\text {ext }}^{(i)}$.

For $\sigma \in \mathscr{E}_{\text {int }}$, we note $\sigma=K \mid L$ for $(K, L) \in \mathscr{M}^{2}$ such that $\partial K \cap \partial L=\sigma$ and we associate the dual cell $D_{\sigma}$ with $D_{K, \sigma} \cup D_{L, \sigma}$, where $D_{K, \sigma}$ (resp. $D_{L, \sigma}$ ) is the halfpart of $K$ (resp. $L$ ) adjacent to $\sigma$. If $\sigma \in \mathscr{E}_{\text {ext }}$ is adjacent to the cell $K$, then $D_{\sigma}=$ $D_{K, \sigma}$. We can define $\Omega$ from the dual mesh: $\Omega=\cup_{\sigma \in \mathscr{E}_{i}} D_{\sigma}, i=1, \ldots, d$. A dual face separating two duals cells $D_{\sigma}$ and $D_{\sigma^{\prime}}$ is denoted by $\varepsilon=\sigma \mid \sigma^{\prime}$. In agreement with the staggered MAC scheme, we will define the unknowns $(\rho, p)$ on the primal grid and the $i-t h$ component of the velocity on $\mathscr{E}^{(i)}$ in such a way that we deal with quantities $\left(\rho_{K}, p_{K}\right)_{K \in \mathscr{M}}$ and $\left(\mathbf{u}_{\sigma}\right)_{\sigma \in \mathscr{E}}$. The grids and notations for $d=2$ are illustrated in Fig. 1.
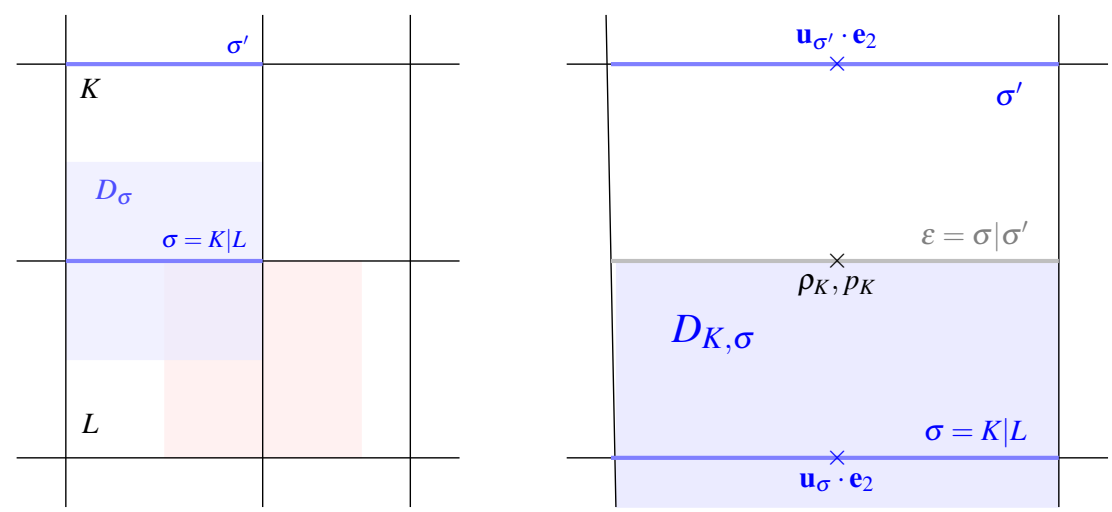

Fig. 1 2-dimensional representation of $(\mathscr{M}, \mathscr{E})$

The discretization of problem (2) begins with the discrete approximation of the mass balance equation (2a). For $K \in \mathscr{M}$, we resort in the classic way to the divergence formula for the computation of (2a) integrated over the primal cell $K$. This yields:

$$
\frac{\rho_{K}^{n+1}-\rho_{K}^{n}}{\delta t}+\frac{1}{|K|} \sum_{\substack{\sigma \in \mathscr{E}(K) \\ \sigma \in \mathscr{E}_{\mathrm{int}}^{(i)}}} F_{K, \sigma}^{n+1}=0,
$$

where $F_{K, \sigma}^{n+1}$ refers to the mass flux across the primal face $\sigma$ outward $K$. In section 2.2 , we introduce the two techniques we adopt to compute $F_{K, \sigma}^{n+1}$; namely the classic upwind scheme and an antidiffusive scheme inspired by [4]. 
Let us focus on the discretization of the prediction step. For $\sigma=K \mid L \in \mathscr{E}_{\text {int }}^{(i)}$, the approximation of (2b) is obtained by integrating the $i-t$ prediction equation over the associated dual cell $D_{\sigma}$. In order for the scheme to be stable and provide the desired estimates for the unknowns, we must pay a particular attention to the discretization of the convective term in (2b):

$$
\frac{1}{\left|D_{\sigma}\right|} \int_{D_{\sigma}}\left(\left(\rho^{n+1} \mathbf{u}^{n+1}-\rho^{n} \mathbf{u}^{n}\right) / \delta t+\nabla \cdot\left(\rho^{n+1} \mathbf{u}^{n+1} \otimes \overline{\mathbf{u}}^{n}\right)\right) \cdot \mathbf{e}_{i} \quad \mathbf{d x}
$$

so that the discretization of the prediction step is compatible with the the discrete mass balance. This yields an approximation of the form:

$$
\left(\rho_{D_{\sigma}}^{n+1} u_{\sigma}^{n+1}-\rho_{D_{\sigma}}^{n} u_{\sigma}^{n}\right) / \delta t+\frac{1}{\left|D_{\sigma}\right|} \sum_{\varepsilon \in \mathscr{E}\left(D_{\sigma}\right)} F_{\sigma, \varepsilon}^{n+1} u_{\varepsilon, i}^{n+1}
$$

where the values for the density on $D_{\sigma}$, denoted $\rho_{D_{\sigma}}$ (resp. the fluxes on the dual faces $\sigma$, noted as $F_{\sigma, \varepsilon}^{n+1}$ ) are given as functions of the density on the primal cells $K, L$ (resp. the fluxes on the primal faces). This is achieved by averaging the discrete mass balance over $K$ and $L$ to obtain a consistent mass balance equation on $D_{\sigma}$. Therefore we define $\left|D_{\sigma}\right| \rho_{D_{\sigma}}^{n}=\left|D_{K, \sigma}\right| \rho_{K}^{n}+\left|D_{L, \sigma}\right| \rho_{L}^{n}$. The dual fluxes $F_{\sigma, \varepsilon}^{n+1}$ are defined as the average of the fluxes on matching primal faces - the primal faces with coinciding outward normals $[10,6]$. The approximation $u_{\varepsilon, i}^{n+1}$ of the $i-t h$ component of the velocity valued on the dual face $\varepsilon$ is obtained by the upwind scheme. Finally the discrete $i-t h$ prediction step is given by

$$
\begin{aligned}
\left(\rho_{D_{\sigma}}^{n+1} u_{\sigma}^{n+1}\right. & \left.-\rho_{D_{\sigma}}^{n} u_{\sigma}^{n}\right) / \delta t+\frac{1}{\left|D_{\sigma}\right|} \sum_{\varepsilon \in \mathscr{E}\left(D_{\sigma}\right)} F_{\sigma, \varepsilon}^{n+1} u_{\varepsilon, i}^{n+1} \\
& -\left(\nabla \cdot\left(\mu^{n+1} \mathbf{D}\left(\mathbf{u}^{n+1}\right)\right)\right)_{D_{\sigma}}+\left(\nabla\left(2 p^{n}-p^{n-1}\right)\right)_{D_{\sigma}}=f_{\sigma}^{n+1}
\end{aligned}
$$

125

with $\left|D_{\sigma}\right| f_{\sigma}^{n+1}=\int_{D_{\sigma}} \mathbf{f}^{n+1} \mathbf{d x}$. The remaining terms are discretized in a straightforward way, with $\left(\nabla\left(2 p^{n}-p^{n-1}\right)\right)_{D_{\sigma}}$ approximated by $\left(|\sigma| /\left|D_{\sigma}\right|\right)\left(\varphi_{L}^{n}-\varphi_{K}^{n}\right) \mathbf{n}_{K, \sigma} \cdot \mathbf{e}_{i}$, if we note $\varphi^{n}=2 p^{n}-p^{n-1}$ and define $\mathbf{n}_{K, \sigma}$ as the normal to the face $\sigma$ outward $K$. For the viscous term, precautions must be taken given the discontinuous nature of the viscosity. It comes down to the discretization of the following term:

$$
-\int_{\partial D_{\sigma}} \mu^{n+1} \mathbf{D}\left(\mathbf{u}^{n+1}\right):\left(\mathbf{n}_{\varepsilon, \sigma} \otimes \mathbf{e}_{i}\right) \mathbf{d} \mathbf{x}
$$

130 Therefore involving the value of $\mu_{\varepsilon}$, the viscosity valued on the faces of $D_{\sigma}$. We refer to [7] for the complete approximation of the viscous term. 


\subsection{Antidiffusive transport scheme for the particles}

For the advection of the density we resort on one hand to the classic upwind scheme, and on the other hand to an antidiffusive transport technique we introduce below. As stated in the previous section, the difference in those methods primarily involves the computation of $F_{K, \sigma}^{n+1}$ in (3).

For the upwind scheme the latter is defined as $F_{K, \sigma}^{n+1}=|\sigma| \rho_{\sigma}^{n+1} \bar{v}_{K, \sigma}^{n}$ with $\bar{v}_{K, \sigma}^{n}=$ $\bar{v}_{\sigma}^{n} \mathbf{n}_{K, \sigma} \cdot \mathbf{e}_{i}$ when $\sigma \in \mathscr{E}^{(i)}$. The updated density at the face $\sigma=K \mid L$, denoted $\rho_{\sigma}^{n+1}$, is given by:

$$
\rho_{\sigma}^{n+1}= \begin{cases}\rho_{K}^{n+1}, & \bar{v}_{K, \sigma}^{n} \geq 0 \\ \rho_{L}^{n+1}, & \text { otherwise }\end{cases}
$$

However in the context of the non-homogeneous Navier-Stokes equations penalized by the H1-penalty method, this approximation of the mass balance generates a large numerical diffusion around the solid phase (as observed in Section 3). We replace the diffusive upwind technique for the transport of the density with an antidiffusive scheme (AD-scheme) based on [2,4] and adapted to the dimension $d=3$ of the problem by considering an alternate directions variant.

Let us focus on the transport of the density by the AD-scheme in the direction $\mathbf{e}_{i}$. For $K \in \mathscr{M}$ we note $\rho_{K}^{*}$ the updated value of the density on $K$ computed from its previous value $\rho_{K}$. Let $\left(K^{-}, K^{+}\right) \in \mathscr{M}^{2}$ so that the primal cells $K^{-}, K, K^{+}$are consecutive and such that $\sigma^{-}=K^{-} \mid K$ and $\sigma^{+}=K \mid K^{+}$are in $\mathscr{E}^{(i)}$. We reorder the cells by imposing $\mathbf{n}_{K, \sigma^{+}} \cdot \mathbf{e}_{i} \geq 0$ and $\mathbf{n}_{K, \sigma^{-}} \cdot \mathbf{e}_{i} \leq 0$. For the time being, Let us assume that the velocities are positive. The transport of the density in $i-t h$ direction is carried out by:

$$
\rho_{K}^{*}=\rho_{K}-\frac{\delta t|\sigma|}{|K|}\left(\left(\rho_{\sigma^{+}} u_{\sigma^{+}}-\rho_{\sigma^{-}} u_{\sigma^{-}}\right)-\left(u_{\sigma^{+}}-u_{\sigma^{-}}\right) \rho_{K}\right)
$$

where $u_{\sigma^{+}}$(resp. $u_{\sigma^{-}}$) is the value of the $i-t h$ component of the velocity on $\sigma^{+}$ ( resp. $\sigma^{-}$). The density on the faces $\sigma^{+}$and $\sigma^{-}$, noted $\rho_{\sigma^{+}}$and $\rho_{\sigma^{-}}$, are to be determined. An equivalent formulation yields:

$$
\rho_{K}^{*}=\rho_{K}+v_{\sigma^{+}}\left(\rho_{K}-\rho_{\sigma^{+}}\right)+v_{\sigma^{-}}\left(\rho_{\sigma^{-}}-\rho_{K}\right)
$$

by defining $v_{\sigma^{+}}=\delta t|\sigma| u_{\sigma^{+}} /|K|$ and $v_{\sigma^{-}}=\delta t|\sigma| u_{\sigma^{-}} /|K|$ as local Courant numbers. We compute the value $\rho_{\sigma^{+}}$in such a way that $\rho_{K}^{*}$ is a convex combination of $\rho_{K^{-}}, \rho_{K}, \rho_{K^{+}}$. Let us note $\llbracket a, b \rrbracket=[\min (a, b), \max (a, b)]$. It then comes down to the projection of the downwind value on $\sigma^{+}\left(\rho_{K^{+}}\right.$in this case) on $\llbracket \rho_{K}, \rho_{K^{+}} \rrbracket \cap \llbracket \rho_{K}, \rho_{K}+\left(1-v_{\sigma^{-}}\right) / v_{\sigma^{+}}\left(\rho_{K}-\rho_{K^{-}}\right) \rrbracket$ using the classic minmod formula. We carry out a similar process for any values of $\mathbf{u}$ on faces $\sigma^{+}, \sigma^{-}$[2]. We extend the advection to other directions to obtain the alternate direction variant of the AD-scheme: 


$$
\begin{gathered}
\rho_{K}^{*}=\rho_{K}-v_{\sigma^{+}}^{(1)}\left(\rho_{K}-\rho_{\sigma^{+}}^{(1)}\right)-v_{\sigma^{-}}^{(1)}\left(\rho_{\sigma^{-}}^{(1)}-\rho_{K}\right) \\
\rho_{K}^{* *}=\rho_{K}^{*}-v_{\sigma^{+}}^{(2)}\left(\rho_{K}^{*}-\rho_{\sigma^{+}}^{*}{ }^{(2)}\right)-v_{\sigma^{-}}^{(2)}\left(\rho_{\sigma^{-}}^{*}{ }^{(2)}-\rho_{K}^{*}\right) \\
\rho_{K}^{* * *}=\rho_{K}^{* *}-v_{\sigma^{+}}^{(3)}\left(\rho_{K}^{* *}-\rho_{\sigma^{+}}^{* *}\right)-v_{\sigma^{-}}^{(3)}\left(\rho_{\sigma^{-}}^{* *}(3)-\rho_{K}^{* *}\right)
\end{gathered}
$$

\section{Numerical Test - Dropping a ball in a viscous fluid}

We drop a rigid heavy sphere in a viscous fluid and observe it reach its terminal velocity according to the principle that the drag force exerted on the particle by the fluid as well as buoyancy balances the gravity applied to the sphere. We define the fluid using the density and viscosity $\rho_{f}=\mu_{f}=1$. The sphere with radius $r=0.08$ and density $\rho_{s}=100$ is falling in the rectangular domain $[0,1] \times[0,1] \times[0,3]$ to which we applied channel-flow boundary conditions. The gravity constant applied to the ball is $g=9.81$. We take for the penalty viscosity $\mu_{s}=10^{4}$. For the time step we will be using $\delta t=0.001$. The spatial step $h$ is such that $h=\max _{i=x, y, z} h_{i}=1 / 50$. The initial position for the ball is $(0.5,0.5,1)$. In this particular test we resort to the upwind scheme for the approximation of fluxes on the primal faces. However this technique produced a large diffusion of the discontinuous quantities and a deformability of the particle (that is ensured by a well-advected viscosity), thus resulting in an incorrect terminal velocity. The advection of the solid phase requires an antidiffusive scheme as introduced in Section 2.2, and complementing numerical tests are on the way.
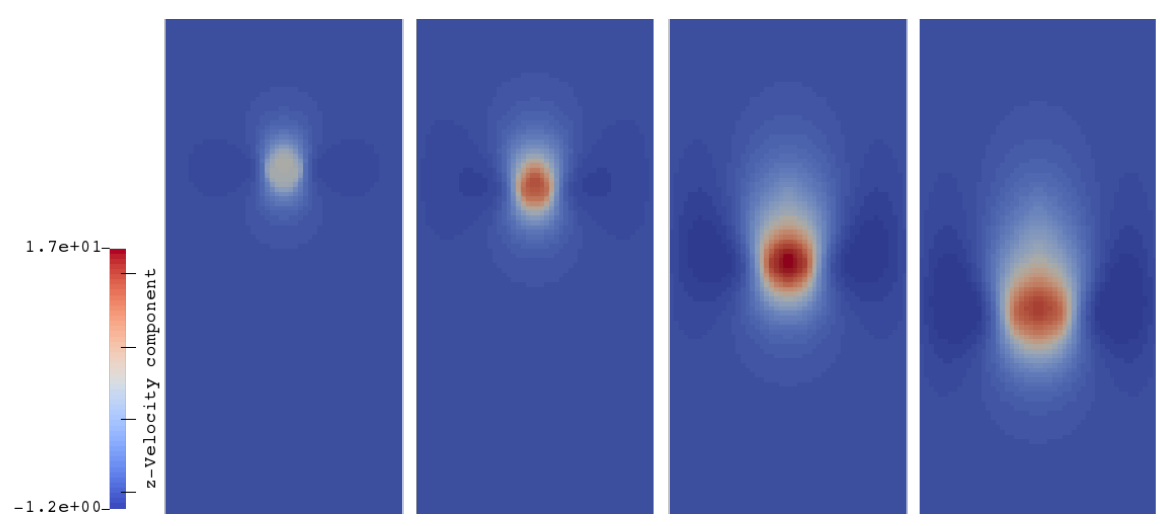

Fig. 2 State of the Velocity component in the Z-direction at times $t=0.01, t=0.02, t=0.05$, $t=0.07$. 


\section{References}

1. Angot, P., Bruneau, C.H., Fabrie, P.: A penalization method to take into account obstacles in incompressible viscous flows. Numer. Math. 81, 497-520 (1999)

2. Bokanowski, O., Zidani, H.: Anti-dissipative schemes for advection and application to Hamilton-Jacobi-Bellmann equations. J. Sci. Comput. 30, 1-33 (2007)

3. Carbou, G., Fabrie, P.: Boundary layer for a penalization method for viscous incompressible flow. Adv. Differential Equ. 8(12), 1453-1480 (2003)

4. Després, B., Lagoutière, F.: Contact discontinuity capturing schemes for linear advection and compressible gas dynamics. J. Sci. Comput. 16(4), 479-524 (2001)

195 5. Févrière, C., Laminie, J., Poullet, P., Angot, P.: On the penalty-projection method for the Navier-Stokes equations with the mac mesh. J. Comput. Appl. Math. 226, 228-245 (2009)

6. Gallouët, Thierry, Gastaldo, Laura, Herbin, Raphaele, Latché, Jean-Claude: An unconditionally stable pressure correction scheme for the compressible barotropic navier-stokes equations. ESAIM: M2AN 42(2), 303-331 (2008)

200

7. Grapsas, D., Herbin, R., Kheriji, W., Latché, J.C.: An unconditionally stable staggered pressure correction scheme for the compressible Navier-Stokes equations. SMAI J. Comput. Math. 2, 51-97 (2016)

8. Guermond, J., Minev, P., Shen, J.: An overview of projection methods for incompressible flows. Comput. Methods Appl. Mech. Engrg. 195(44), 6011 - 6045 (2006)

9. Guermond, J.L., Salgado, A.: A splitting method for incompressible flows with variable density based on a pressure poisson equation. J. of Comput. Phys. 228(8), $2834-2846$ (2009)

10. Herbin, R., Latché, J.C.: Kinetic energy control in the MAC discretization of compressible Navier-Stokes equations. Int. J. Fin. Vol 7(2) (2010)

11. Hoffmann, K.H., Starovoitov, V.: On a motion of a solid body in a viscous fluid. twodimensional case. Adv. Math. Sci. Appl. 9, 633-648 (1999) 